\title{
Optimization of Non-Orthogonal Multiple Access Based Visible Light Communication Systems
}

\author{
Zanib Tahira, Hafiz M. Asif ${ }^{(}$, Asim Ali Khan, Sobia Baig $^{\circledR}$, Shahid Mumtaz ${ }^{\circledR}$, and Saba Al-Rubaye ${ }^{(\mathbb{1}}$
}

\begin{abstract}
In visible light communication (VLC), the data is transmitted by modulating the light emitting diode (LED). The data-rate is throttled by the narrow modulation bandwidth of LEDs, which becomes a barrier for attaining high transmission rates. Non-orthogonal multiple access (NOMA) is a new scheme envisioned to improve the system capacity. In addition to multiple access schemes, optimization techniques are applied to further improve the data rate. In this letter, convex optimization is applied to NOMA-based VLC system for downlink. The proposed optimization system is analyzed in terms of the bit error rate (BER) and the sum-rate.
\end{abstract}

Index Terms-Visible light communication (VLC), nonorthogonal multiple access (NOMA), fifth generation (5G), nonconvex optimization, multiobjective optimization, Cuckoo Search algorithm.

\section{INTRODUCTION}

$\mathbf{T}$ HE emergence of the Fifth-Generation (5G) service based applications, such as virtual reality and augmented reality, has increased the demand for huge chunks of bandwidth to meet users demand. Therefore, new radio access interfaces are employed to support gigabit throughput with milliseconds latency. Among others, VLC is a promising technology that offers a huge licensed-free spectrum, low latency, immunity to interference from electromagnetic sources, low-power consumption, no security issues and less harmful compared with Radio Frequency (RF) modules [1]. Due to its unique characteristics, VLC is considered as the most suitable and secure alternative to RF communications especially for niche applications such as positioning [2]. A typical VLC system incorporates certain systematic features supporting high capacity, high Signal-to-Noise-Ratio (SNR), reduced Bit Error Rate (BER), and optimized received power. To enable such characteristics, 5G adopts disruptive technologies such as millimeter wave (mmWave) and Non-Orthogonal Multiple Access (NOMA) schemes to serve VLC users with improved spectral efficiency [3]. Recently, there were numerous works analyzing the use of mmWave and NOMA for VLC. For example,

Manuscript received September 7, 2018; revised October 30, 2018 and December 10, 2018; accepted December 12, 2018. Date of publication January 1, 2019; date of current version August 12, 2019. The associate editor coordinating the review of this letter and approving it for publication was $\mathrm{T}$. Ngatched. (Corresponding author: Hafiz M. Asif.)

Z. Tahira, H. M. Asif, A. A. Khan, and S. Baig are with the Department of Electrical and Computer Engineering, COMSATS University Islamabad, Lahore Campus, Lahore 54000, Pakistan (e-mail: zanibtahira@ gmail.com; hafizasif@cuilahore.edu.pk; drasim@cuilahore.edu.pk; drsobia@ cuilahore.edu.pk).

S. Mumtaz is with GS-Lda, 351-96-1178227 Aveiro, Portugal (e-mail: shmu@gs-lda.com).

S. Al-Rubaye is with the School of Aerospace, Transport and Manufacturing, Cranfield University, Bedford MK43 0AL, U.K. (e-mail: s.alrubaye@cranfield.ac.uk).

Digital Object Identifier 10.1109/LCOMM.2018.2889986
H. Marshoud et al. proposed using optical-NOMA technique to solve the problem of LEDs low modulation bandwidth within 5G state-of-the-art techniques [4]. The articles [4] and [5] proposed various power allocation schemes to improve the data rate, i.e., gain ratio power allocation (GRPA) and normalized GRPA of NOMA. Along with the above studies, some other works used optimization techniques to multiply the VLC capacity. For instance, the capacity of multiple-input multipleoutput NOMA (MIMO-NOMA) was found to be higher than MIMO orthogonal multiple access (MIMO-OMA) considering sum-channel capacity and ergodic sum-capacity parameters [6]. In [7], a non-convex single objective optimization problem was proposed to maximize sum-throughput under fairness and light intensity constraints. In [8], authors proposed a power allocation strategy for sum-rate maximization in NOMAbased VLC systems under power control and quality-of-service constraints. In a slightly different approach, a multiobjective optimization problem was used for VLC to quantify the conflict between the received power and SNR in [9].

Most state of the art works on VLC are using deterministic numerical methods of optimization such as interior point method, gradient descent method, linear programming, and Lagrangian Relaxation method. These optimization methods have proven to be infeasible for practical non-convex multiobjective problems, i.e., at times, they lead to suboptimal solutions. In the last two decades, nature-inspired metaheuristic optimization methods have been proposed for constrained, complex, non-convex and multiobjective optimization problems. Specifically, Cuckoo Search (CS) is one of the most efficient algorithms among other metaheuristic optimization algorithms [10]. However, few works are found on multiobjective problems and their optimal solutions for NOMAVLC system. Therefore, in this letter, a novel and realistic optimization problem is formulated as a multiobjective nonconvex function. The novelty of the work lies in optimizing two key parameters of NOMA-VLC, i.e., the capacity and the received power (which in turn improves SNR) as twofold advantage. Then, the optimization problem is solved as non-convex problem using the CS algorithm and without employing convex problem analysis. Intensive simulations are also carried out to validate the given solution for the optimization problem.

The rest of the letter proceeds as follows: Section II presents the system model and problem formulation of the multiobjective optimization. Section III describes the solution of the multiobjective optimization through a nature-inspired CS Algorithm. Section IV compares simulation results of the conventional system with the optimized system, followed by conclusion in the last section. 


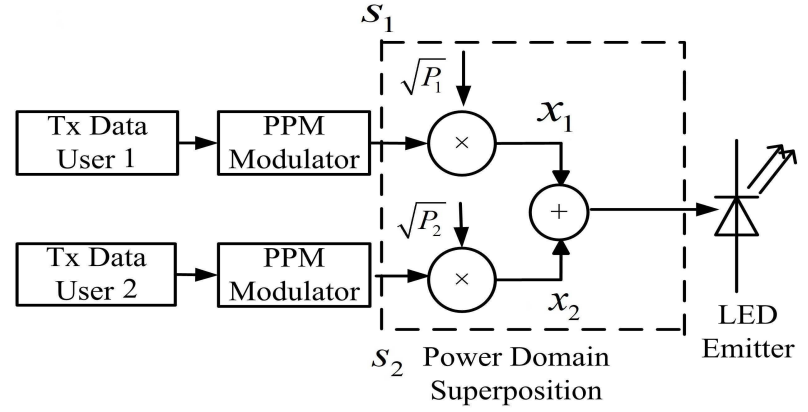

Fig. 1. Block diagram of NOMA-based VLC system.

\section{System Model and Problem Formulation}

\section{A. System Model}

The proposed system model considers NOMA downlink system with superposition transmission and successive interference cancellation (SIC) at the user terminals. The superposition coding technique is used for signal transmission, while SIC multi-user detection is employed to detect the desired signals at the receiver side. In addition, the system considers a realistic scenario with a single LED acting as transmitter, and two photodiodes (PDs) acting as two users (User \#1 and User \#2), as shown in Fig. 1. NOMA enables the LED transmitter to connect with the users over the same frequency band of radio resource. Accordingly, both users are associated or connected to the LED, since they are located within the coverage of the LED beam at different distances. According to the NOMA principle, different power levels $p_{1}$ and $p_{2}$ on the same subcarrier are associated with User \#1 and User \#2, respectively. We assume that User \#1 is the Far user while User \#2 is the Near user, based on the distance from the transmitter. Therefore, the corresponding transmitted signal is represented by [7]:

$$
\mathbf{x}=\sum_{m=1}^{2} \sqrt{p_{m}} s_{m}+A .
$$

where $s_{m}$ is the message signal of user $m$ and $p_{m}$ is the power allocated to user $m . A$ is the direct current (DC) offset/bias that is added to ensure the positive instantaneous intensity of the transmitted signal, and $\mathbf{x}$ is the linear combination of data for the two users. An 8-ary Pulse Position Modulation (PPM) scheme is used to modulate the data. The constraints for non-negativity of transmitted signal and the intensity values under allowed peak optical intensity $B$ are similar to [7]. In order to maintain non-negativity of the transmitted signal $s_{m}$, the following constraint has to be satisfied:

$$
\sum_{m=1}^{2} \sqrt{p_{m}} \leq A
$$

To consider eye safety, the transmitted optical intensity should be limited by the peak optical intensity $B$, as in the following constraint

$$
\sum_{m=1}^{2} \sqrt{p_{m}} \leq B-A
$$

The superimposed signal is received after passing through the VLC channel of the receiver with additional independent zero-mean additive white Gaussian noise (AWGN) noise $n_{m}$, whereas the constant DC-offset is being removed. Let $h_{m}$ be the channel gain between the transmitter and user $m$, with the channel gains arranged in ascending order and the total bandwidth normalized to unity. Therefore, the received signal at user $m$ is given as

$$
y_{m}=\mathbf{s} h_{m}+n_{m}
$$

where $\mathbf{s}=\sum_{m=1}^{2} \sqrt{p_{m}} s_{m}(\mathbf{s}=\mathbf{x}-A)$ is the linear combination of the users data after removing the DC offset. For NOMA, a user with lower channel gain implements SIC technique to decode its signal. Since more power is assigned to User \#1 (Far user), data is decoded directly, while User \#2 employs the SIC algorithm [11].

\section{B. Multiobjective Problem Formulation}

In this section, we present a multiobjective problem for NOMA-VLC to maximize the two key objectives of sumrate and received power. A weighted sum approach is used to convert the problem into a single objective maximization problem for these two objective functions:

$$
\begin{aligned}
& \max _{\boldsymbol{p}, \psi_{c}, \Phi_{1 / 2}, T(\psi)}\left[w _ { 1 } \sum _ { m = 1 } ^ { 2 } \operatorname { l o g } _ { 2 } \left(\log _{2}(1\right.\right. \\
& \left.\left.\left.+\frac{h_{m}^{2} p_{m}}{h_{m}^{2} \sum_{l=m+1}^{2} p_{l}+\sigma^{2}}\right)\right)+w_{2} \sum_{m=1}^{2} p_{m} h_{L o S}(0)\right] \\
& \text { s.t. } \sum_{m=1}^{2} p_{m} \leq P_{\text {max }} \\
& \quad \sum_{m=1}^{2} \sqrt{p_{m}} \leq C \\
& \quad \boldsymbol{p} \geq 0 \\
& \quad 30^{\circ} \leq \Psi_{c} \leq 80^{\circ} \\
& 15^{\circ} \leq \Phi_{1 / 2} \leq 90^{\circ} \\
& 1.5 \leq T(\psi) \leq 4
\end{aligned}
$$

where $w_{1}$ and $w_{2}$ are the weights of the multiobjective problem, such that $w_{1}+w_{2}=1$ and $h_{L o S}(0)=$ $\frac{A_{r} R\left(\phi_{T_{i}}\right)}{d^{2}} T_{s}(\psi) g(\psi) \cos (\psi)$ refers to the DC gain of the line of sight (LoS) VLC channel, whereas $h_{L o S}(t)$ represents LoS 
TABLE I

Optimization PRoblem PARAMETERS

\begin{tabular}{lll}
\hline Parameter & Description & Value \\
\hline$p$ & Power vector & $\boldsymbol{p}=\left(p_{1}, p_{2}\right)^{T}$ \\
$C$ & Intensity limit & $C=\min \{A, B-A\}$ \\
$R\left(\phi_{T_{x_{i}}}\right)$ & Radiant intensity & $R\left(\phi_{T_{x_{i}}}\right)=\frac{(k+1)}{2 \pi} \cos ^{k}\left(\phi_{T_{x_{i}}}\right)$ \\
$k$ & Lambertian emission mode & $k=\frac{-\ln 2}{\ln \left(\cos \Phi_{1 / 2}\right)}$ \\
$g(\psi)$ & Optical lens gain & $g(\psi)=\frac{n^{2}}{\sin ^{2} \Psi_{c}}$ \\
\hline
\end{tabular}

components of the transmission channel [9]. The objective function with weight $w_{1}$ represents the sum-rate of the VLC system. The objective function with weight $w_{2}$ is the received power of users in the VLC system. $P_{\max }$ is the maximum power of the transmitter. This last constraint is necessary to make sure that the total power allocated to the users does not exceed the maximum value, as shown in (5b) and (5c), respectively. What is so beautiful about the NOMA solutions is their remarkable flexibility, since they have a host of different parameters, which allow us to appropriately configure them to meet the Quality of Service (QoS) requirements.

The most important system-level parameters include $\phi_{T_{x_{i}}}$, which represents the irradiance angle of the LED, and $\Phi_{1 / 2}$, which is the LED's semi-angle at half-power (5f). $A_{r}$ denotes the surface area of the PD, where $d$ is the distance between the LED and PD. $\psi$ indicates the incidence angle of the PD and $T_{s}(\psi)$ is the gain of the optical filter $(5 \mathrm{~g}) . n$ is the refractive index and $\Psi_{c}$ is the field-of-view (FOV) of the lens. The additional parameters considered in the system are given in Table I. Moreover, constraints (5e)-(5g) represent the channel parameters that can vary on a specific range to maximize the received power of PDs/users to optimize the channel for maximum efficiency. It is important to note that these constraints are also used in the CS algorithm to maximize the sum-rate and received power of the users.

\section{Problem Solution}

The formulated objective function is non-convex due to the non-convexity of the objective function and constraints defined in (5a) and (5c), (5e), (5f), respectively. The optimization problem is solved through CS algorithm [12]. This algorithm is based on breeding behavior of certain species of cuckoo. The CS algorithm in combination with Lévy flights, is more efficient than genetic algorithms and particle swarm optimization because of fewer parameters. It is more generic and robust compared to other metaheuristic algorithms and potentially more powerful to solve multiobjective optimization problems with large number of constraints; it can even solve NP-hard problems. First of all, the population of host nests (parameters of objective function) is initialized and the stopping criterion is configured, then a solution is randomly initialized by the Lévy flights and its fitness is evaluated under fitness criteria. The new solution is replaced by the previous one depending upon fitness, and this process continues on generating new solutions and keeping the best one, until it finds the best values for objective function. Using this approach, the worse nests
TABLE II

Simulation PARAMETERS

\begin{tabular}{ll}
\hline Parameter & Value \\
\hline Room size & $4 \mathrm{~m} \mathrm{x} \mathrm{4m} \mathrm{x} \mathrm{2m}$ \\
Modulation order & 8 -ary PPM \\
Channel type & VLC channel LoS \\
Noise type & AWGN \\
Receiver's height & $1.5 \mathrm{~m}$ \\
PD's responsitivity & $0.45 \mathrm{~A} / \mathrm{W}$ \\
Area of PD & $1 \mathrm{~cm}^{2}$ \\
SNR range & $0-10 \mathrm{~dB}$ \\
Max. transmitted power & $12 \mathrm{~mW}$ \\
\hline
\end{tabular}

are abandoned and new and better solutions are kept, while choosing the best one [12]. The pseudo code of CS with Lévy flights is shown in Algorithm 1 [13].

\section{A. Simulation Parameters}

In this section, we provide details about the MATLAB simulation environment for optimized NOMA-based VLC system. The simulations are performed using CS algorithm with maximum number of iteration $=2 \times 10^{3}$, number of nests/solutions $=25$, discovery rate of alien eggs $=0.25$ and Lévy flight exponent, $\beta=3 / 2$. The system parameters include $w_{1}=0.7, w_{2}=0.3$ (more weight for Obj1 than $O b j 2$ ), $A=20 \sqrt{d B m}, B=30 \sqrt{d B m}$ and the noise power spectral density $=10^{-13} \mathrm{~m} W^{2} / H z$. The parameter values for the VLC channel are listed in Table II.

\section{Simulation Results}

The conventional NOMA-based VLC system is compared with the optimized NOMA-based VLC system considering BER, sum-rate and received power, as shown in Fig. 2, Fig. 3 and Fig. 4, respectively. For all simulations, fixed power allocation scheme has been used for assigning power to the users. The total power is $12 \mathrm{~mW}$, where $9 \mathrm{~mW}$ is assigned to User \#1 and $3 \mathrm{~mW}$ to User \#2.

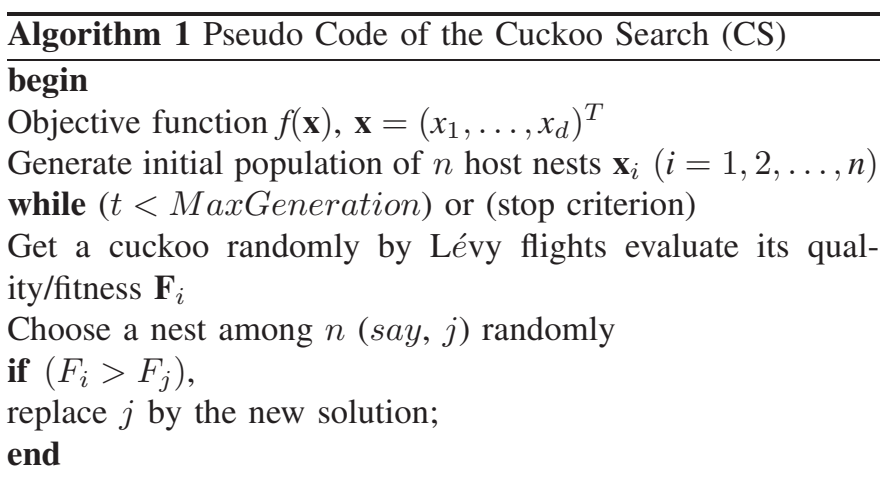

A fraction $\left(p_{a}\right)$ of worse nests are abandoned and new ones are built; Keep the best solutions

(or nests with quality solutions);

Rank the solutions and find the current best

end while

Postprocess results and visualization end 


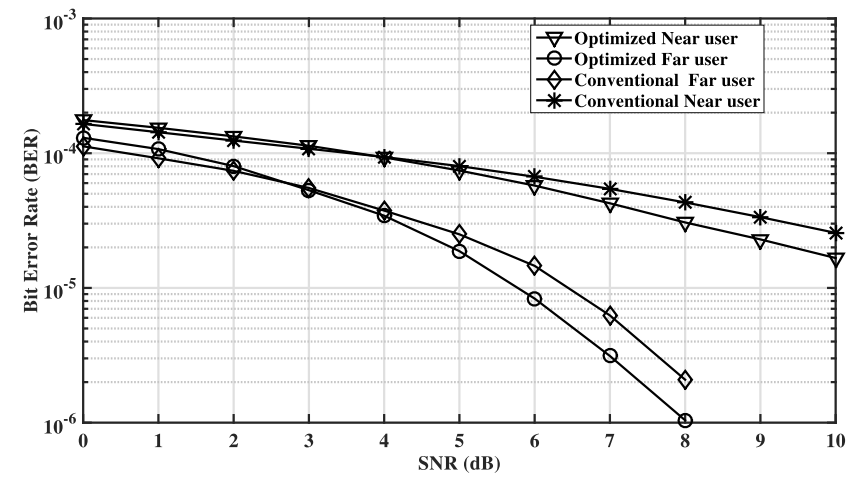

Fig. 2. BER vs. SNR for the conventional and optimized NOMA-based VLC systems.

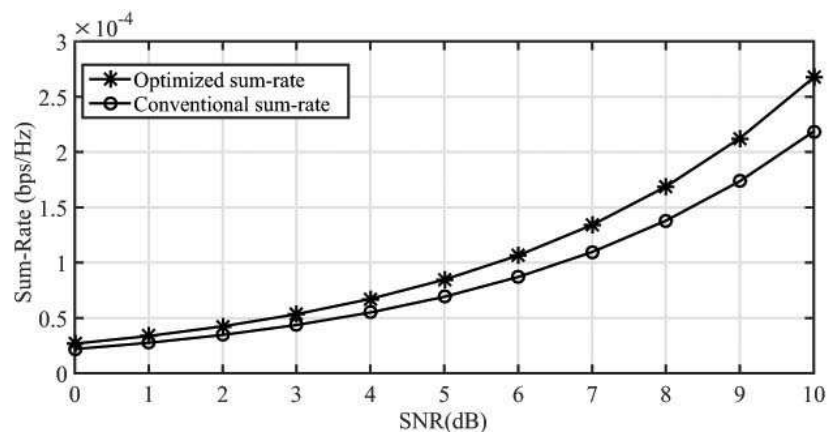

Fig. 3. Sum-rate vs. SNR for the conventional and optimized NOMA-based VLC systems.

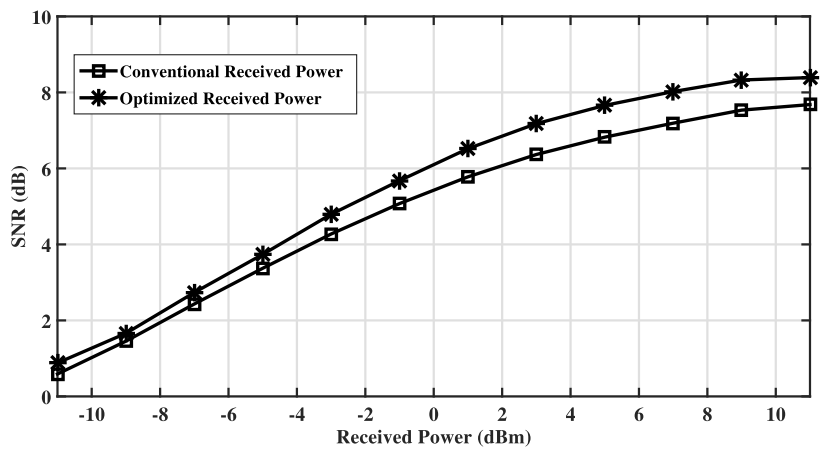

Fig. 4. Received power vs. SNR for the conventional and optimized NOMA-based VLC systems.

For the optimized NOMA system, the non-convex multiobjective problem is solved through the CS algorithm; it maximizes the sum-throughput of the system by assigning optimized power values to the users. The optimum power values provided by the CS algorithm for User \#1 and User $\# 2$ are $p_{1}=7.89 \mathrm{~mW}$ and $p_{2}=3.92 \mathrm{~mW}$, respectively. In addition, the optimized values of the channel parameters are: FOV of the lens is $\Psi_{c}=57.55$, LED's semi-angle at half-power is $\Phi_{1 / 2}=46.70$ and the gain of the optical lens is $T(\psi)=3.48$. Fig. 2 show the BER curves for both users, while the sum-rate improvements are calculated by taking the percentage difference of their conventional and optimized values. To this end, the SNR values are preconfigured for various simulation iterations. For $8 d B$ SNR, the BER improvement is calculated as $49.99 \%$ for optimized User \#1 and $28.99 \%$ for optimized User \#2, as shown in Fig. 2. For $8 d B$ SNR, the sum-rate improvement is $18.25 \%$ for the optimized system, as shown in Fig. 3. For $7 d B$ SNR, the improvement in the received power of users is calculated as $58.33 \%$ for the optimized system, as shown in Fig. 4. The simulation results show that the BER of the NOMA-based VLC has been reduced through the optimization technique, while the sum-rate and the received power of the users in the system have been maximized.

\section{CONCLUSION}

In this letter, a promising multiple access technology based on sum-rate and received power maximization optimization is considered. A downlink NOMA-based VLC system scheme was presented using optimal and computationally efficient optimization techniques, while considering the user fairness and unique intensity constraints. The simulation results have shown that the optimized NOMA-based VLC system outperforms the conventional NOMA-based VLC system in terms of BER, the sum rate and the received power. In future work, the proposed optimization technique can be reformulated for other wireless (non-VLC) channels.

\section{REFERENCES}

[1] M. Ahsan and H. M. Asif, "ESIM-OFDM-based transceiver design of a visible light communication system," Int. J. Commun. Syst., vol. 30, no. 8, May 2017, Art. no. e3175. [Online]. Available: http://onlinelibrary. wiley.com/doi/10.1002/dac.3175/full

[2] A. Naz, H. M. Asif, T. Umer, and B.-S. Kim, "PDOA based indoor positioning using visible light communication," IEEE Access, vol. 6, pp. 7557-7564, 2018.

[3] K. Chandra, A. S. Marcano, S. Mumtaz, R. V. Prasad, and H. L. Christiansen, "Unveiling capacity gains in ultradense networks: Using mm-wave NOMA," IEEE Veh. Technol. Mag., vol. 13, no. 2, pp. 75-83, Jun. 2018.

[4] H. Marshoud, P. C. Sofotasios, S. Muhaidat, G. K. Karagiannidis, and B. S. Sharif, "On the performance of visible light communication systems with non-orthogonal multiple access," IEEE Trans. Wireless Commun., vol. 16 , no. 10 , pp. 6350-6364, Oct. 2017.

[5] C. Chen, W.-D. Zhong, H. Yang, and P. Du, "On the performance of MIMO-NOMA-based visible light communication systems," IEEE Photon. Technol. Lett., vol. 30, no. 4, pp. 307-310, Feb. 15, 2018.

[6] M. Zeng, A. Yadav, O. A. Dobre, G. I. Tsiropoulos, and H. V. Poor, "Capacity comparison between MIMO-NOMA and MIMO-OMA with multiple users in a cluster,' IEEE J. Sel. Areas Commun., vol. 35, no. 10, pp. 2413-2424, Oct. 2017.

[7] Z. Yang, W. Xu, and Y. Li, "Fair non-orthogonal multiple access for visible light communication downlinks," IEEE Wireless Commun. Lett., vol. 6, no. 1, pp. 66-69, Feb. 2017.

[8] H. Shen, Y. Wu, W. Xu, and C. Zhao, "Optimal power allocation for downlink two-user non-orthogonal multiple access in visible light communication," J. Commun. Inf. Networks, vol. 2, no. 4, pp. 57-64, Dec. 2017.

[9] Z. Ghassemlooy et al., Optical Wireless Communications: System and Channel Modelling with MATLAB. Boca Raton, FL, USA: CRC Press, 2017.

[10] P. M. Pardalos, A. Žilinskas, and J. Žilinskas, Non-convex Multiobjective Optimization. Springer, 2017.

[11] H. Marshoud, S. Muhaidat, P. C. Sofotasios, S. Hussain, M. A. Imran, and B. S. Sharif, "Optical non-orthogonal multiple access for visible light communication," IEEE Wireless Commun., vol. 25, no. 2, pp. 82-88, Apr. 2018.

[12] X.-S. Yang, Nature-inspired Metaheuristic Algorithms. Luniver Press, 2010.

[13] X.-S. Yang and S. Deb, "Cuckoo Search via Lévy flights," in Proc. World Congr. Nature Biologically Inspired Comput. (NaBIC), Dec. 2009, pp. 210-214. 
2019-01-01

\section{Optimization of non-orthogonal multiple access based visible light communication systems}

Tahira, Zanib

IEEE

Tahira Z, Asif HM, Khan AA, et al., Optimization of non-orthogonal multiple access based visible light communication systems. IEEE Communications Letters, Volume 23, Issue 8, 2019, pp. 1365-1368 https://doi.org/10.1109/LCOMM.2018.2889986 Downloaded from Cranfield Library Services E-Repository 